\title{
Needs in omega 3 and ocular pathologies
}

\author{
Lionel BRETILLON ${ }^{1}$ \\ Emilie SIMON $^{1}$ \\ Niyazi ACAR ${ }^{1}$ \\ Olivier BERDEAUX ${ }^{2}$ \\ Alain BRON ${ }^{1,3}$ \\ Catherine CREUZOT-GARCHER ${ }^{1,3}$ \\ ${ }^{1}$ Eye, Nutrition \& Signalling Research \\ Group, \\ Centre des Sciences du Goût et de \\ I'Alimentation, \\ UMR 1324 Inra, \\ 6265 CNRS, \\ Université de Bourgogne, \\ Centre Inra, \\ 17 rue Sully, \\ BP 86510, \\ 21065 Dijon cedex, \\ France \\ $<$ Lionel.bretillon@dijon.inra.fr> \\ ${ }^{2}$ ChemoSens Platform, \\ Centre des Sciences du Goût et de \\ I'Alimentation, \\ UMR 1324 Inra, \\ 6265 CNRS, \\ Université de Bourgogne, \\ Dijon, \\ France \\ ${ }^{3}$ Department of Ophthalmology, \\ University hospital, \\ Dijon, \\ France
}

The past decades have been characterized by the improvement of life expectancy and changes of the living including dietary habits of the Western populations. Meanwhile, the development of pathologies has emerged. Eye diseases remain the second most prevalent ones

\begin{abstract}
Life expectancy at birth has regularly increased decade after decade, especially since the beginning of the 20th century: 15 years have been gained over the past 50 years. Changes in living and dietary habits during this time period have been associated with the development of various pathologies which represent a growing socioeconomic burden. Among age-related disorders, ocular diseases are the second most prevalent ones after 65 years. Age-related Macular Degeneration (AMD) is the leading cause of visual impairment after the age of 50 years. Age is the prominent risk factor for $A M D$ and is accompanied with both endogenous (including genetics) and environmental factors, such as smoking habits and dietary factors (diet rich in cholesterol and saturated fatty acids). AMD is characterized by the loss of cells at the most central area of the retina, called macula. The neural retina is a highly structured neurosensory tissue that is responsible for the transduction pathway. The transduction pathway is initiated in photoreceptors where the light stimulus is coded into an electrical signal. This signal is transmitted to neighboured neurons and transferred to the brain via the optic nerve. The retinal pigment epithelium (RPE) is the cellular and metabolic interface between the neural retina and choriocapillaris through Bruch's membrane. The close association between RPE and photoreceptors is one of the factors that promote the efficacy of RPE to, in the one hand, provide nutrients and oxygen to photoreceptors and, in the other hand, eliminate the metabolic debris originating from shedding of the outer segments. Epidemiological data suggest that dietary habits privileging the consumption of omega3 long chain polyunsaturated fatty acids participate to prevent from the development of AMD (Sangiovanni et al., 2009). The mechanisms underlying the effects of omega-3 fatty acids remain unclear until now. The purpose of the present paper is to give a review on the role, metabolism and effects of omega 3 fatty acids in the retina.
\end{abstract}

Key words: lipid, omega 3, retina, nutrition, aging, prevention

after the age of 65 years in Western countries. Accounting to the demographic forecasts, patients with eye diseases are expected to represent a sensitive and growing socio-economic burden. Aging remains one of the most influencing factors associated with the development of retinal pathologies. Age-related Macular Degeneration (AMD) is the leading cause of visual impairment of the aged developed populations. Environmental factors, including dietary habits, are also of some concerns in the development of AMD. Aging of the retina is characterized by specific clinical, functional and morphological features, including lipid deposition. Lipids are quantitatively important components of the retina but their roles are not fully defined. Lipids may both promote and prevent aging of the retina. The purpose of this review is to highlight the roles and benefits of lipids and dietary fatty acids in aging and age-related diseases.

\section{Lipids are structural components of the retina}

The term "retina" encompasses both the neural retina and the retinal pigment epithelium (RPE). The retina covers the internal part of the ocular globe at its posterior pole (figure 1).

To cite this article: Bretillon L, Simon E, Acar N, Berdeaux O, Bron A, Creuzot-Garcher C. Needs in omega 3 and ocular pathologies. OCL 2011; 18(5): 279-283. doi : 10.1684/ocl.2011.0407 
The neural retina is a neurosensory tissue which primary function is to convert light photons into an electrical signal. This function is called the transduction pathway. The neurosensory retina contains photosensitive cells (rods and cones), neurons and glial cells. Various types of neurons are present in the neurosensory retina: bipolar cells, ganglion cells, amacrine cells, horizontal cells (figure 1). The architecture of the neurosensory retina is reverse to the way light enters. Cones and rods are located at the most external side of the neurosensory retina, at the vicinity of the RPE. The coding function of the retina is dependent not only to photoreceptors but also to neurons, glial cells and RPE which amplify the signal. Rods represent the prominent population of photoreceptors compared to cones, even in most diurnal animal species (Masland, 2001). The structural organization of photoreceptors and neurons in the retina is unique. The signal emerges from rods and cones independently, is transmitted to bipolar cells, converges to ganglion cells, and is transferred to the brain via the optic nerve. On the contrary to the cone pathway which involves a one-toone association of cone-bipolar cellganglion cell, the rod system is much more convergent since the signal from many rods is pooled to generate a signal

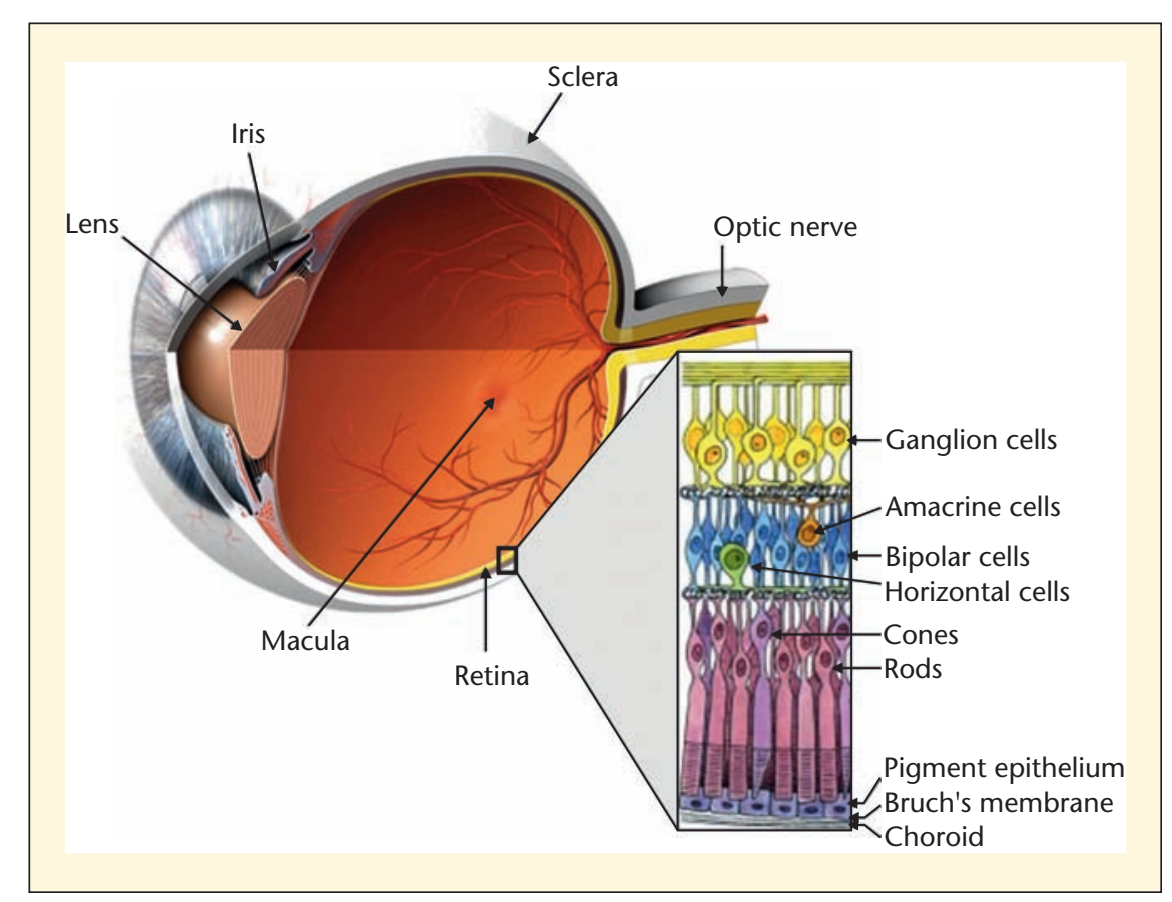

Figure 1. Structure of the human eye and organization of the retina

in one ganglion cells. About 100 millions of cones and rods and 1 million of ganglion cells are present in the retina. This relationship between photoreceptors, bipolar cells and ganglion cells maximizes the response to light, especially in rods. The ability of photoreceptors to convert light photons into an electrical signal is due to the presence of a photopigment (opsin in cones, rhodopsin in rods) in their outer segments. The outer segment of a photoreceptor consists in a stack of disk membranes (figure 2) that are synthesized in the proximal portion of the outer segment (close to inner segment, B-panel in figure 2), and shed at its apical side by the RPE (C-panel in figure 2). RPE forms villi that entrap the outer segments of the photoreceptors, and thereby improves the capacity of the RPE to eliminate the debris, and provide the neuroretina with nutrients.

Rhodopsin is a G-protein coupled receptor which is present in the outer segments. Absorption of photons by rhodopsin yields conformational movements of rhodopsin that result in activation of the G-protein and biological response. The lipid environment of rhodopsin is a key effector of these changes (Brown et al., 2010). The neurosensory retina is composed of $90 \%$ phospholipids and $10 \%$ cholesterol (Bretillon et al.,
2008; Fliesler and Bretillon, 2010). Docosahexaenoic acid (DHA) is a long chain polyunsaturated fatty acid (LC-PUFA) from the omega 3 series. It is present at high levels in the neurosensory retina: about $15 \%$ in the whole human retina (Bretillon et al., 2008), and accounts for $50 \%$ of the fatty acids in the outer segments of photoreceptors (Fliesler and Anderson, 1983). DHA improves the kinetics of the photocycle by creating specific inter-molecular associations with rhodopsin. The highly unsaturated chemical structure of DHA with six double bonds confers enhanced fluidity to DHArich membranes which ameliorates their biophysical parameters. On the contrary, saturated fatty had opposite effects (Litman and Mitchell, 1996); cholesterol stabilizes rhodopsin and impairs rhodopsin activation (Grossfield et al., 2006). Electroretinography is a suitable method to monitor the capacity of the retina to respond to light stimulus. The retina of animals reared under a diet deficient in omega 3 fatty acids is depleted in DHA, and shows a reduced electroretinographic response (Neuringer et al., 1986). In addition to DHA, very LC-PUFA with 32 or 34 atoms of carbon are found in the human retina (Berdeaux et al., 2010). Their function remains uncertain but their deficiency is associated with a specific retinal phenotype including impaired electroretinographic response, increased accumulation of a toxic vitamin A derivative and degeneration of photoreceptor cells in the central retina (Agbaga et al., 2008; Karan et al., 2005). These features are associated with mutations in the gene coding ELOVL4 (elongation of very long chain fatty acids 4) and with the dominantly inherited juvenile macular degeneration called Stargardt-like macular dystrophy (STGD3) in humans (Karan et al., 2005). The interested reader should refer to the review from Berdeaux in the present issue of the journal.

\section{Where do fatty acids in the retina come from?}

The capacity of the retina to get enriched in DHA from dietary sources is relatively low. Bazan reported two decades ago that DHA is recycled with high efficiency in the outer segments and is also provided by circulating sources (Bazan, 1989). But the relative contribution of exogenous sources and recycling to retinal DHA remains poorly 


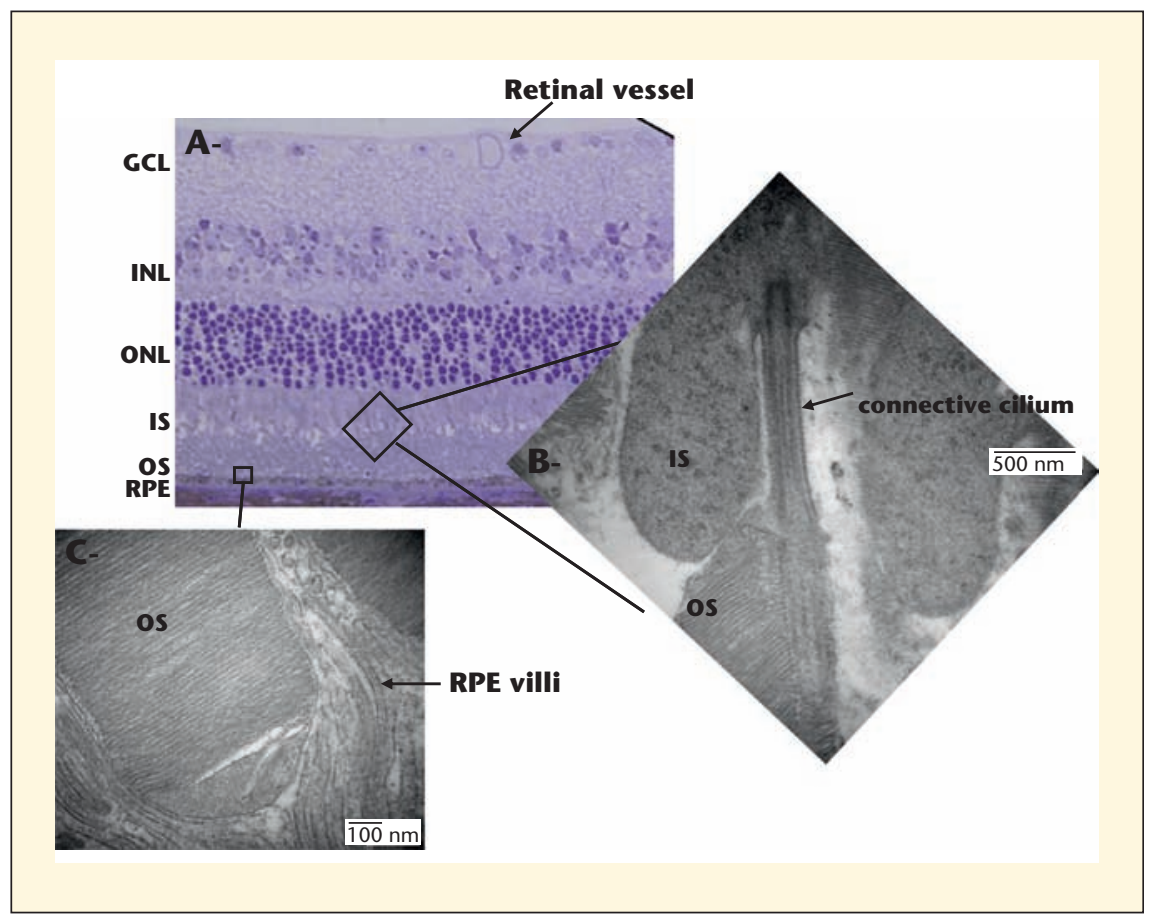

Figure 2. Morphological structure of the whole (A-panel) and outer retina (B-and C-panels)

defined. Recently, we have analyzed retinal and adipose tissue samples from human donors. The fatty acid profile of adipose tissue was considered as a surrogate for long term history intake in dietary fatty acids of the subjects. Linoleic acid is exclusively of dietary origin. Its content in the neurosensory retina was strongly and positively associated with its adipose tissue level. On the contrary, no similar association was observed with DHA levels (Bretillon et al., 2008). Therefore, we suggest that only a small portion, at best, of retinal DHA may be derived from dietary DHA. The efficacy of dietary supplementation with DHA to enhance the accumulation of DHA in the neurosensory retina remains unknown in humans. Data in laboratory animals favour a positive although relatively minor effect. Indeed, using long term supplementations with omega 3 LC-PUFA (19.2\% of the dietary fatty acids), DHA concentration increases in the retina only by less than $10 \%$, compared to a control diet devoid of omega 3 LC-PUFA (Schnebelen et al., 2009; Schnebelen et al., 2009).

Circulating fatty acids are found esterified with a glycerol backbone in triglycerides and phospholipids, and linked to cholesterol in cholesteryl esters. Triglycerides are the major lipid components of chylomicrons and VLDL which are lation is a clinical sign of maculopathy: the early stage of AMD. The composition of drusen has not been fully determined, but include lipofuscin, fibrillar and nonfibrillar amyloid, cholesterol, glycoproteins, vitronectin, inhibitors and activators of the extracellular matrix, complement factor $\mathrm{H}$, complement component $\mathrm{C} 3$, and zinc. This accumulation of debris creates a lipid wall which participates to the age-associated thickening of Bruch's membrane and to the increase in hydraulic resistance. Such accumulation of lipid-rich particles within Bruch's membrane may reduce the fluxes of nutrients to the retina, and may be involved in the partial loss of retinal functionality in a relevant rodent model of aging of the human retina: the transgenic mouse expressing the human apolipoprotein $B_{100}$ and lacking LDLreceptor (Bretillon et al., 2008).

AMD targets a specific area of the retina: the macula (figure 1). Visual field of AMD patients is characterized by the loss of central vision. AMD patients therefore poorly discriminate colors and details. Aging, genetic and environmental factors participate to the development of AMD, advanced age being the prominent one. High fat intake, and especially saturated fatty acids and cholesterol, has been associated with higher risk for AMD (Age-Related Eye Disease Study Research Group, 2007). Advanced stages of AMD are of two types: wet or dry AMD. Wet AMD, also called neovascular AMD, affects about $40 \%$ of the population with late AMD. It is characterized by choroidal neovascularization, whereas the primary clinical characteristic of dry AMD is the appearance of RPE atrophy, also called geographic atrophy. The clinical management of patients with neovascular AMD is of peculiar interest given the incidence of choroidal neovascularisation, and potentially intraretinal haemorrhages. Drug therapies and laser treatments are so far gold standards for clinical ophthalmologists in AMD patients. Preventive approaches would also be pertinent in patients with maculopathy.

AREDS (Age-Related Eye Disease Study) is a multicenter study funded by the National Institutes of Health in the USA. This natural history study and phase III clinical trial was designed to assess the clinical course, prognosis, risk factors, and nutrient-based prevention and treatment of AMD. More than 4700 
participants were initially enrolled from November 1992 to January 1998. The 5 -years follow-up was completed in April 2001, and continued until December 2005 to evaluate the 4-years clinical course and progression of AMD for participants previously enrolled in the trial. Numerous reports have been published so far on this trial. The last one reveals that participants who have the highest omega 3 LC-PUFA intake $(0.11 \%$ of total energy intake) were $30 \%$ less likely to develop geographic atrophy and neovascular AMD than lower consumers $(0.01 \%$ of total energy intake) (Sangiovanni et al., 2009). The Blue Mountains Eye Study in Australia has reported similar association: one serving of fish per week was associated with reduced risk of early AMD by $30 \%$, primarily in subjects with less than the median linoleic acid consumption (Tan et al., 2009). The US Twin Study of AMD was derived from the National Academy of Sciences-National Research Council World War II Veteran Twin Registry. This registry is the largest population-based twin registry in the US and includes information for 15924 white male twin pairs born between 1917 and 1927 who served in the US armed forces. The large size of this population gave the unique opportunity to evaluate the role of genetic and environmental risk factors for age-related diseases including AMD. Data from 681 twins report that two or more servings of fish per week reduced the risk of AMD by 2-fold. This reduction in risk was seen primarily among subjects with low levels of linoleic acid intake (Seddon et al., 2006).

The mechanisms behind this protection are poorly defined. We recently questioned whether following the epidemiological-based guidelines (increase omega 3 LC-PUFAs and reduce linoleic acid intake) would enhance the enrichment of the neurosensory retina and RPE with omega 3 LC-PUFAs, and modulate gene expression in the neurosensory retina (Simon et al., 2011). Diets rich in omega 3 LC-PUFAs efficiently improve the incorporation of omega 3 LC-PUFAs in the tissues. This raising effect was magnified by lowering linoleic acid intake. Reducing linoleic acid intake up-regulated the expression of genes coding for transporters of lipids and enzymes involved in lipid metabolism (LDL-receptor, CD36, ABCA1, ALOX5 and ALOX12). LDLR, $A B C A 1$ and $C D 36$ have been suggested to participate to lipid recycling in the neurosensory retina (Tserentsoodol et al., 2006; Tserentsoodol et al., 2006). ALOX5 and ALOX12 are lipoxygenase enzymes that catalyze the hydroxylation of PUFAs. DHA may be converted into such biologically active metabolites in the RPE (Bazan et al., 2010). Neuroprotectin D1 is a stereospecific derivative of DHA, produced after the release of DHA from phospholipids and hydroxylation by 15-lipoxygenase. NPD1 is a cell mediator which activates pro-survival repair signalling. NPD1 exhibits anti-inflammatory properties, induces anti-apoptotic proteins and inhibits pro-apoptotic proteins. Thus, NPD1 triggers activation of signalling pathway that promotes cell survival. NPD1 would be of peculiar importance in the response of RPE cells to oxidative stress during photoreceptor outer segment phagocytosis and in the course of AMD (Bazan et al., 2010).

\section{Fatty acids and glaucoma}

Glaucoma is the second leading cause of blindness worldwide. More than 60 millions of glaucoma patients are expected in 2020 in the world (Quigley, 2011). Glaucoma is a progressive optic neuropathy which is characterized by the loss of retinal ganglion cells (figure 1).Various risk factors have been associated with glaucoma, such as high intraocular pressure, age, familial history, ethnicity, gene polymorphisms, and myopia (Quigley, 2005). Epidemiological data reported that major fats and lipids were not associated with glaucoma (Kang et al., 2004). But higher ratio of omega 3 to omega 6 LC-PUFA was positively associated with a greater risk of glaucoma $(+50 \%)$, especially in subjects with elevated intra-ocular pressure (Kang et al., 2004). Animal data showed that omega 3 LC-PUFA deficiency impaired the electroretinographic response of retinal ganglion cells (Nguyen et al., 2008), and increased intra-ocular pressure (Nguyen et al., 2007). Recently, we published that not only dietary omega 3 LC-PUFA, but also the combination of omega 3 LC-PUFA and omega 6 fatty acids, modulate the stress of the retina to elevated intra-ocular pressure in early hours (Schnebelen et al., 2011) and at long term (Schnebelen et al., 2009). Interestingly, we found that glaucoma patients had erythrocytes with reduced levels of DHA-rich phospholipids and plasmalogens. The differences were associated with the severity of glaucoma (Acar et al., 2009).

\section{Conclusion}

Lipids represent the most energetic nutrients. In Western and developed populations, more than one third of the daily energy intake comes from lipids. Dietary recommendations for fatty acid intake to the French population have recently been re-evaluated. Omega 3long chain polyunsaturated fatty acids (EPA and DHA) are now included not only in order to fulfil the physiological needs but also in order to prevent from agerelated disorders, including AMD. Oxidative stress and inflammation are with certainty the most influencing mechanisms in the aging processes. In addition to their role as fuels, lipids are also metabolic substrates and cellular effectors that intervene in those various cellular mechanisms.

\section{REFERENCES}

Acar N, Berdeaux O, Juaneda $\mathrm{P}$, et al. Red blood cell plasmalogens and docosahexaenoic acid are independently reduced in primary open-angle glaucoma. Exp Eye Res 2009; 89: 840-53.

Agbaga MP, Brush RS, Mandal MNA, Henry $\mathrm{K}$, Elliott MH, Anderson RE. Role of Stargardt3 macular dystrophy protein (ELOVL4) in the biosynthesis of very long chain fatty acids. Proc Natl Acad Sci U S A 2008; 105: 12843-8.

Age-Related Eye Disease Study Research Group. The Relationship of Dietary Lipid Intake and Age-Related Macular Degeneration in a Case-Control Study: AREDS Report No. 20. Arch Ophthalmol 2007; 125: 671-9.

Bazan N. Inherited retinal degeneration. In: M. LaVail, R. Anderson, and J. Hollyfield (Eds), Inherited and environmentally induced retinal degenerations. Elsevier: New York, 1989.

Bazan NG, Calandria JM, Serhan CN. Rescue and repair during photoreceptor cell renewal mediated by docosahexaenoic acid-derived neuroprotectin D1. J Lipid Res 2010; 51: 2018-31.

Berdeaux O, Juaneda P, Martine L, Cabaret S, Bretillon L, Acar N. Identification and quantification of phosphatidylcholines containing very long chain polyunsaturated fatty acid (VLC-PUFA) in bovine and human retina by liquid chromatography/tandem mass spectrometry. J Chromatogr A 2010; 1217: 7738-48.

Bretillon L, Acar N, Seeliger $\mathrm{MW}$, et al. $\mathrm{ApoB}_{100}, \mathrm{LDLR}^{-/-}$mice exhibit reduced elec- 
troretinographic response and cholesteryl esters deposits in the retina. Invest Ophthalmol Vis Sci 2008; 49: 1307-14.

Bretillon L, Thuret G, Grégoire S, et al. Lipid and fatty acid profile of the retina, retinal pigment epithelium/choroid, and lacrimal gland, and associations with adipose tissue fatty acids in human subjects. Exp Eye Res 2008; 87: 521-8.

Brown MF, Salgado GFJ, Struts AV. Retinal dynamics during light activation of rhodopsin revealed by solid-state NMR spectroscopy. Biochim Biophys Acta 2010; 1798: 177-93.

Curcio CA, Johnson M, Huang JD, Rudolf M. Aging, Age-related Macular Degeneration, and the Response-to-Retention of Apolipoprotein B-Containing Lipoproteins. Progr Retin Eye Res 2009; 28: 393-422.

Fliesler SJ, Anderson RE. Chemistry and metabolism of lipids in the vertebrate retina. Prog Lipid Res 1983; 22: 79-131.

Fliesler S], Bretillon L. The ins and outs of cholesterol in the vertebrate retina. / Lipid Res 2010; 51: 3399-413.

Grossfield A, Feller SE, Pitman MC. A role for direct interactions in the modulation of rhodopsin by \{omega\}-3 polyunsaturated lipids. Proc Natl Acad Sci USA 2006: 48884893.

Kang JH, Pasquale LR, Willett WC, et al. Dietary fat consumption and primary openangle glaucoma. Am / Clin Nutr 2004; 79: 755-64.

Karan G, Lillo C, Yang Z, et al. Lipofuscin accumulation, abnormal electrophysiology, and photoreceptor degeneration in mutant ELOVL4 transgenic mice: a model for macular degeneration. Proc Natl Acad Sci U S A 2005; 102: 4164-9.

Litman BJ, Mitchell DC. A role for phospholipid polyunsaturation in modulating membrane protein function. Lipids 1996; 31 (Suppl. 31): S193-7.
Masland $\mathrm{RH}$. The fundamental plan of the retina. Nat Neurosci 2001; 4: 877-86.

Neuringer, M., Connor, W.E., Lin, D.S., Barstad, L., Luck, S. Biochemical and functional effects of prenatal and postnatal omega 3 fatty acid deficiency on retina and brain in rhesus monkeys. Proc Natl Acad Sci U S A 1986; 83: 4021-5.

Nguyen CT, Vingrys AJ, Bui BV. Dietary omega-3 fatty acids and ganglion cell function. Invest Ophthalmol Vis Sci 2008; 49: 3586-94.

Nguyen CTO, Bui BV, Sinclair AJ, Vingrys AJ. Dietary Omega 3 Fatty Acids Decrease Intraocular Pressure with Age by Increasing Aqueous Outflow. Invest Ophthalmol Vis Sci 2007; 48: 756-62.

Quigley HA. Glaucoma: macrocosm to microcosm the friedenwald lecture. Invest Ophthalmol Vis Sci 2005; 46: 2663-70.

Quigley HA. Glaucoma. The Lancet 2011; 377: 1367-77.

Sangiovanni JP, Agron E, Meleth AD, et al. \{omega\}-3 Long-chain polyunsaturated fatty acid intake and 12-y incidence of neovascular age-related macular degeneration and central geographic atrophy: AREDS report 30, a prospective cohort study from the AgeRelated Eye Disease Study. Am J Clin Nutr 2009; 90: 1601-7.

Schnebelen C, Fourgeux C, Pasquis B, et al. Dietary polyunsaturated fatty acids reduce retinal stress induced by an elevation of intraocular pressure in rats. Nutrition Research 2011; 31: 286-95.

Schnebelen $\mathrm{C}$, Grégoire $\mathrm{S}$, Pasquis $\mathrm{P}$, et al. Dietary n-3 and n-6 PUFA enhances DHA incorporation in retinal phospholipids without affecting PGE1 and PGE2 levels. Lipids 2009; 44: 465-70.

Schnebelen C, Pasquis B, Salinas-Navarro M, et al. A dietary combination of omega-3 and omega- 6 polyunsaturated fatty acids is more efficient than single supplementations in the prevention of retinal damage induced by elevation of intraocular pressure in rats. Graefes Arch Clin Exp Ophthalmol 2009; 247: 1191-203.

Schnebelen C, Viau S, Grégoire S, et al. Nutrition for the eye: different susceptibility of the retina and the lacrimal gland to dietary omega- 6 and omega- 3 polyunsaturated fatty acid incorporation. Ophthalmic Res 2009; 41: 216-24.

Seddon JM, George S, Rosner B. Cigarette smoking, fish consumption, omega-3 fatty acid intake, and associations with agerelated macular degeneration: the US Twin Study of Age-Related Macular Degeneration. Arch Ophthalmol 2006; 124: 995 1001.

Simon E, Bardet B, Gregoire S, et al. Decreasing dietary linoleic acid promotes long chain omega-3 fatty acid incorporation into rat retina and modifies gene expression. Exp Eye Res 2011; doi:10.1016/j.exer.2011.07.016.

Tan JSL, Wang JJ, Flood V, Mitchell P. Dietary fatty acids and the 10-year incidence of agerelated macular degeneration: the blue mountains eye study. Arch Ophthalmol 2009; 127: 656-65.

Tserentsoodol N, Gordiyenko NV, Pascual I, Lee JW, Fliesler SJ, Rodriguez IR. Intraretinal lipid transport is dependent on high density lipoprotein-like particles and class B scavenger receptors. Mol Vis 2006; 12: 1319-33.

Tserentsoodol N, Sztein J, Campos M, et al. Uptake of cholesterol by the retina occurs primarily via a low density lipoprotein receptor-mediated process. Mol Vis 2006; 12: 1306-18. 